\title{
Associated risk factors with disease severity and antiviral drug therapy in patients with COVID-19
}

\author{
Xiaowei Gong ${ }^{1}$, Shiwei Kang ${ }^{1}$, Xianfeng Guo ${ }^{2}$, Yan $\mathrm{Li}^{1}$, Haixiang Gao ${ }^{3}$ and Yadong Yuan ${ }^{1 *}$ (D)
}

\begin{abstract}
Background: Due to the latent onset of novel coronavirus disease 2019 (COVID-19), it is important to identify patients with increased probabilities for disease progression early in order to implement timely medical strategies. This study aimed to identify the factors associated with increased COVID-19 severity and evaluate the current antiviral drugs, especially in severe patients.

Methods: This was a retrospective observational study performed at the No. 7 Hospital of Wuhan (Wuhan, China) with hospitalized patients confirmed with COVID-19 from January 11 to March 13, 2020. Multivariable logistic regression analysis was used to identify the associated factors of severe COVID. Treatments of antivirus drugs were collected and evaluated.

Results: Of the 550 patients, 292 (53.1\%) were female and 277 (50.4\%) were $\geq 60$ years old. The most common symptom was fever $(n=372,67.7 \%)$, followed by dry cough $(n=257,46.7 \%)$, and dyspnea $(n=237,43.1 \%)$, and fatigue $(n=224$, 40.7\%). Among the severe patients, $20.2 \%$ required invasive ventilator support and $18.0 \%$ required non-invasive ventilator. The identified risk factors for severe cases were: age $\geq 60$ years (odds ratio $(\mathrm{OR})=3.02,95 \%$ confidence interval $(\mathrm{Cl}): 1.13-8.08$, $P=0.028)$, D-dimer $>0.243 \mu \mathrm{g} / \mathrm{ml}(\mathrm{OR}=2.734,95 \% \mathrm{Cl}: 1.012-7.387, P=0.047)$, and low oxygenation index $(\mathrm{OR}=0.984,95 \% \mathrm{Cl}$ : $0.980-0.989, P<0.001$ ). In severe cases, the benefits (relief of clinical symptoms, clinical outcome, and discharge rate) of arbidol alone was $73.3 \%$, which was better than ribavirin $(7 / 17,41.2 \%, P=0.029)$.
\end{abstract}

Conclusions: Age $\geq 60$ years, D-dimer $>0.243 \mu \mathrm{g} / \mathrm{ml}$, and lower oxygenation index were associated with severe COVID-19. Arbidol might provide more clinical benefits in treating patients with severe COVID-19 compared with ribavirin.

Keywords: COVID-19, SARS-CoV-2, Disease severity, Associated factors, Antiviral drug

\section{Background}

A novel member of the coronavirus family, severe acute respiratory syndrome coronavirus 2 (SARS-CoV-2), has infected more than 42 million people in the world as of October 27, 2020, since it was first identified in December 2019, causing over 1.1 million deaths [1-7]. SARS$\mathrm{CoV}-2$ is found on all continents and in nearly all

\footnotetext{
*Correspondence: yuanyd1108@163.com

1 Department of Respiratory and Critical Care Medicine, The Second Hospital of Hebei Medical University, Shijiazhuang 050000, China

Full list of author information is available at the end of the article
}

countries [6]. On March 11, 2020, the World Health Organization (WHO) announced that the coronavirus disease 2019 (COVID-19) had become a pandemic [8]. Currently, the domestic epidemic in China has been largely controlled after a painful nation-wide war against COVID-19. Unfortunately, this epidemic occurred when our knowledge about similar previous viruses, the acute respiratory syndrome coronavirus (SARS-CoV) and Middle East respiratory syndrome coronavirus (MERS-CoV), was still limited. This lack of knowledge severely limited the response against the virus and its spread.

C C The Author(s). 2021 Open Access This article is licensed under a Creative Commons Attribution 4.0 International License, which permits use, sharing, adaptation, distribution and reproduction in any medium or format, as long as you give appropriate credit to the original author(s) and the source, provide a link to the Creative Commons licence, and indicate if changes were made. The images or other third party material in this article are included in the article's Creative Commons licence, unless indicated otherwise in a credit line to the material. If material is not included in the article's Creative Commons licence and your intended use is not permitted by statutory regulation or exceeds the permitted use, you will need to obtain permission directly from the copyright holder. To view a copy of this licence, visit http://creativecommons.org/licenses/by/4.0/ The Creative Commons Public Domain Dedication waiver (http://creativecommons.org/publicdomain/zero/1.0/) applies to the data made available in this article, unless otherwise stated in a credit line to the data. 
Indeed, the SARS-CoV-2 exhibits 79.5\% homology with SARS-CoV, and patients infected with either of them have similar symptoms, but SARS-CoV-2 is more contagious than SARS-CoV, as shown by an increased reproduction number $\left(\mathrm{R}_{0}\right)$ [9]. Complicating screening, control, and management, the clinical symptoms of COVID-19 are non-specific (fever, cough, and shortness of breath) and are shared by a number of respiratory infections. In addition, many patients are asymptomatic, most of those with symptoms have a good prognosis, and about $20 \%$ of symptomatic patients may experience disease progression and reach a critical condition [10]. Such patients will quickly progress to acute respiratory distress syndrome (ARDS), respiratory failure, multiple organ failure (MODS), or even death [1-5]. Suspected risk factors for severe COVID-19 include age $\geq 65$ years, residence in long-term care facilities, and underlying conditions such as chronic lung disease, a serious heart condition, severe obesity, diabetes, chronic kidney disease, liver disease, and immunocompromising conditions [11].

The virus is still spreading relentlessly and exponentially around the world and creating an enormous threat to global health, with the fear of second and later waves [6]. Unfortunately, no specific therapeutic drugs are yet available. Currently, the treatment of severe and critical cases involves support treatments aiming at maintaining oxygenation and controlling inflammation and coagulation $[12,13]$. Drugs such as hydroxychloroquine have been tried against COVID-19 but finally was not proven effective $[14,15]$. Antiviral drugs might reduce infection duration and time to symptom resolution [16-19]. A study showed that patients receiving remdesivir recovered 5 days faster than those receiving placebo, while $86 \%$ of severe patients recovered an average of 7 days faster [20]. Favipiravir was not associated with the viral clearance rate but with a reduced time to defervescence [21]. Similar benefits were suggested for interleukin (IL)6 inhibitors [22, 23]. Nevertheless, no drug is universally recognized to be effective against COVID-19 [22].

Besides, COVID-19 could elapse as much as 2-3 weeks between virus exposure and symptom onset, and could result in severe or even fatal complications [1-5]. The early identification of patients with an increased likelihood of disease progression is important in order to implement timely medical strategies and to adjust them according to the evolving conditions, especially in the context of the exhausted healthcare systems around the world. Given the limited data on the risk factors for severe patients and effectiveness of antiviral drugs, we determined to identify the clinical factors associated with severe COVID-19 and evaluate the current antiviral drugs, especially in patients with severe COVID-19.

\section{Methods}

\section{Study design and patients}

This single-center, retrospective, observational study was performed at the No. 7 Hospital of Wuhan (Wuhan, China), which is a designated hospital to treat patients with COVID-19. The medical team from the Second Hospital of Hebei Medical University was appointed by the government to provide medical assistance to the No. 7 Hospital of Wuhan during the outbreak. All hospitalized patients diagnosed with "viral pneumonia" from January 11 to March 13, 2020, were preliminarily included in this study. Patients confirmed with COVID-19 were then enrolled in the study. The diagnosis was made following the Chinese COVID-19 management guideline (versions 3 to 7) [12]. Patients with atypical clinical symptoms or chest radiology changes combined with negative SARS-CoV-2 RNA test results were excluded from this study. The study was approved by the Institutional Ethics Board of the No. 7 Hospital of Wuhan and the Second Hospital of Hebei Medical University (Code: 2020-R016). The need for individual consent was waived due to the non-interventional and retrospective nature of this study.

\section{Data collection}

The patients' electronic medical records, which had been archived onto a local server, including epidemiology parameters, clinical presentation, laboratory results, imaging characteristics, treatments, and disease outcomes, were collected by a group of designated physicians who accepted sufficient training. Several important time points were also analyzed, including disease onset, time from disease onset to dyspnea, time for SARS-CoV-2 RNA to be no longer detectable in patients with positive RNA results upon hospital admission, and average hospital stay. For patients who required mechanical ventilation, time from disease onset to ARDS and time to mechanical ventilation were analyzed. All data were checked by two physicians (XWG and SWK). Major disagreement between two physicians was resolved by consultation with a third reviewer (YDY). Any missing or uncertain records were obtained and clarified through direct communication with attending doctors and their families.

\section{Definition}

The exposure history denote that a person in close contact with diagnosed patients or recent visiting of COVID-19 designated hospitals within 2 weeks before the onset of respiratory symptoms. Fever was defined as axillary temperature of at least $37.3^{\circ} \mathrm{C}$. Acute myocardial injury was diagnosed if serum levels of cardiac biomarkers (eg, troponin $\mathrm{T}$ ) were above the 99th percentile upper reference limit, or if new abnormalities were 
shown in electrocardiography and echocardiography [1]. Secondary infection was diagnosed after the occurrence of hospital-acquired pneumonia or bacteremia, plus a positive result of new pathogen culture from the blood and lower respiratory tract specimens (including sputum, bronchoalveolar lavage fluid or tracheal aspirate) after admission [24]. Acute respiratory distress syndrome (ARDS) was diagnosed according to the Berlin Definition [25]. Acute kidney injury was diagnosed according to the KDIGO clinical practice [26]. Shock and organ failure was defined in accordance with the Chinese COVID-19 management guideline [12]. DIC was diagnosed according to ISTH criteria [27].

Disease onset was defined as the time of patients starting to present symptoms. The severe cases were identified according to the Chinese COVID-19 management guideline (versions 3 to 7) [12]. Disease progression was identified and classified when the patients had one of the following criteria: 1) respiratory distress with respiratory frequency $\geq 30 / \mathrm{min}$; 2 ) pulse oximeter oxygen saturation $\leq 93 \%$ at rest; and 3) oxygenation index (artery partial pressure of oxygen/inspired oxygen fraction, $\mathrm{PaO} 2 / \mathrm{FiO} 2) \leq 300 \mathrm{mmHg}$ [12]. Disease improvement was defined as: patients' situation remained unchanged; severe cases changed to non-severe cases; and patients were permitted for discharge. The discharge criteria were: body temperature returned to normal and maintained for more than three consecutive days; significantly improved respiratory symptoms; a significant improvement on imaging and a negative result on RNA tests with two consecutive sputum samples or nasopharyngeal swabs or other respiratory samples (at least $24 \mathrm{~h}$ between each sampling) [12].

\section{Laboratory tests}

The pharynx swabs of suspected patients were collected and transported to the clinical laboratory of Zhongnan Hospital of Wuhan University for RNA detection following strict standard procedures. The presence of SARSCoV-2 in pharynx swabs was detected by real-time RTPCR. The detailed analysis and detection processes can be found in a previous study [3]. Laboratory tests and radiologic assessments, including chest X-ray or computed tomography $(\mathrm{CT})$, were performed on the basis of the state of illness.

\section{Treatment}

Treatment was provided according to the Chinese COVID-19 management guideline (versions 3 to 7) [12], combined with the clinical characteristics of the patients and the actual situation of the medical resources of No. 7 Hospital of Wuhan during the epidemic. Patients with a mild condition were given general support like resting in bed, supportive treatment, antiviral treatment, and antibiotics if necessary. Severe patients were given respiratory and other organs support treatment on an individualized basis. We comprehensively evaluated the treatment effect of the patients by closely observing their clinical condition change and disease outcome.

\section{Statistical analysis}

Categorical variables were presented as frequencies and percentages and analyzed using the chi-square test or Fisher's exact test, as appropriate. Continuous variables were presented as mean \pm standard deviations or medians (interquartile range (IQR)) according to the results of the Kolmogorov-Smirnov test and analyzed using Student's t-test or the Mann-Whitney U-test, according to the distribution. Variables were first screened with univariable logistic regression; variables with $P$-values $<0.05$ for association with severe COVID-19 were included in a multivariable logistic regression analysis. In univariable analysis, we chose a total of 19 variables which had been commonly observed in severe or non-surviving patients [1-5]. Variables were excluded if their $P$-values $>0.05$, if their accuracy could not be confirmed (symptom, which was self-reported), if they were unavailable under emergency circumstances (radiographic findings, troponin $\mathrm{T}$, glomerular filtration rate), if the sample size was relatively small (diabetes, heart disease), if they might be related to other variables (sex, leucocytes, procalcitonin). Besides, considering the earlier analysis of the total number of deaths $(n=52)$ in this study and to avoid model overfitting, the six variables with the strongest association were selected for the multivariable logistic regression analysis on the basis of previous findings and clinical constraints. All statistical analysis was performed using SPSS 22.0 (IBM, Armonk, NY, USA). Two-sided (except for the chi-square test) $P$-values $<0.05$ were considered statistically significant.

\section{Patient and public involvement}

This was a retrospective case series study, and no patients were involved in the study design or in setting the research questions or the outcome measures directly. No patients were asked to advise on the interpretation or writing up of results.

\section{Results}

\section{Demographic and clinical characteristics}

All hospitalized patients $(n=644)$ diagnosed with "viral pneumonia" from January 11 to March 13, 2020, were screened for inclusion. Finally, 550 patients diagnosed with COVID-19 (including 422 cases positive for SARSCoV-2 RNA and 128 cases clinically diagnosed but with negative RNA tests) were included. Table 1 presents the characteristics of the patients. Among all patients, 292 (53.1\%) patients were female, and 258 (46.9\%) were 
Table 1 Demographics and comorbidities of patients with COVID-19

\begin{tabular}{|c|c|c|c|c|}
\hline Variables & All Patients $(n=550)$ & Severe $(n=178)$ & Non-severe $(n=372)$ & $p$ value \\
\hline \multicolumn{5}{|l|}{ Age, years, n (\%) } \\
\hline$<44$ & $103(18.7 \%)$ & $13(7.3 \%)$ & $90(24.2 \%)$ & $<0.001$ \\
\hline $45-59$ & $170(30.9 \%)$ & $36(20.2 \%)$ & $134(36.0 \%)$ & $<0.001$ \\
\hline$\geq 60$ & $277(50.4 \%)$ & $129(72.5 \%)$ & $148(39.8 \%)$ & $<0.001$ \\
\hline \multicolumn{5}{|l|}{ Sex, n (\%) } \\
\hline Female & $292(53.1 \%)$ & $74(41.6 \%)$ & $218(58.6 \%)$ & $<0.001$ \\
\hline Male & $258(46.9 \%)$ & $104(58.4 \%)$ & $154(41.4 \%)$ & $<0.001$ \\
\hline \multicolumn{5}{|l|}{ Source of transmission, n (\%) } \\
\hline None & $342(62.2 \%)$ & $144(80.9 \%)$ & $198(53.2 \%)$ & $<0.001$ \\
\hline Contact history with diagnosed patients & $170(30.9 \%)$ & $22(12.4 \%)$ & $148(39.8 \%)$ & $<0.001$ \\
\hline Recent visit of COVID-19 designated hospitals & $38(6.9 \%)$ & $12(6.7 \%)$ & $26(7.0 \%)$ & $<0.001$ \\
\hline \multicolumn{5}{|l|}{ Comorbidity, n (\%) } \\
\hline Hypertension & $184(33.5 \%)$ & $81(45.5 \%)$ & $103(27.7 \%)$ & $<0.001$ \\
\hline Diabetes & 77 (14.0\%) & $37(20.8 \%)$ & $40(10.8 \%)$ & 0.002 \\
\hline Cardiovascular disease & $56(10.2 \%)$ & $26(14.6 \%)$ & $30(8.1 \%)$ & 0.018 \\
\hline Malignancy & $23(4.2 \%)$ & $13(7.3 \%)$ & $10(2.7 \%)$ & 0.011 \\
\hline Cerebrovascular disease & $20(3.6 \%)$ & $10(5.6 \%)$ & $10(2.7 \%)$ & 0.086 \\
\hline Chronic pulmonary disease & $18(3.3 \%)$ & 7 (3.9\%) & $11(3.0 \%)$ & 0.547 \\
\hline Chronic liver disease & $15(2.7 \%)$ & $4(2.2 \%)$ & $11(3.0 \%)$ & 0.843 \\
\hline Hyperlipidemia & $10(1.8 \%)$ & $5(2.8 \%)$ & $5(1.3 \%)$ & 0.229 \\
\hline
\end{tabular}

Data are shown as median (IQR) or $\mathrm{n}(\%)$. ARDS Acute respiratory distress syndrome, CMV Cytomegalovirus. EBV Epstein-Barr virus

male. Most patients were $>60$ years; $277(50.4 \%)$ and $342(62.2 \%)$ reported no history of exposure to COVID19. Hypertension $(n=184,33.5 \%)$, diabetes $(n=77$, $14.0 \%)$, cardiovascular disease $(n=56,10.2 \%)$, and malignancy $(n=23,4.2 \%)$ were the most frequent comorbidities. Ultimately, 178 patients progressed to a severe condition (32.4\%), and 52 died (9.5\%).

The most common symptom upon diagnosis was fever $(n=372,67.6 \%)$, most frequently between 38 and $39^{\circ} \mathrm{C}$ $(n=204,37.1 \%)$ (Table 2). It is important to point out that a significant portion of patients $(n=178,32.3 \%)$ did not have fever at diagnosis. The remaining common symptoms were dry cough $(n=257,46.7 \%)$, dyspnea $(n=237,43.1 \%)$, fatigue $(n=224,40.7 \%)$, sputum production $(n=169,30.7 \%)$, and abdominal pain/diarrhea $(n=75,13.6 \%)$. Most patients $(n=393,71.5 \%)$ presented with more than one symptom, but only 130 (23.6\%) showed the classical triple signs of COVID-19 (fever, cough, and dyspnea).

The median time from disease onset to admission was 9 (IQR, 6-14) days, the time from disease onset to dyspnea was 0 (IQR, 0-7) days. In this cohort, 69 patients eventually developed ARDS and needed mechanical ventilation. The average time from disease onset to ARDS was 10 (IQR, 6-15) days among patients who eventually developed ARDS, and the mean time to mechanical ventilation was 10 (IQR, 6-15) days in the same subgroup.
Compared with the patients who did not progress to a severe condition, the severe patients were generally older and had a higher proportion of males $(n=104,58.4 \%$ vs. $41.4 \%, P<0.001)$. Patients with clear exposure histories were more often non-severe $(P<0.001)$, while the exposure history was not traceable in most severe patients $(P<0.001)$. For clinical symptoms, most of the nonsevere patients did not have a fever upon hospital admission $(n=157,42.3 \%, P<0.001)$. Patients presenting with moderate or severe fever were more likely to have disease progression $(P<0.001)$, defined as when the illness turned to more severe or critical conditions or death. In addition, dyspnea, fatigue, chill, sputum production, and tachycardia were more common in severe patients. Severe patients were frequently associated with multiple clinical symptoms, especially the classic triple signs $(n=$ $76,42.7 \%, P<0.001$ ) (Table 2).

\section{Laboratory and imaging findings}

Upon hospital admission, all patients underwent relevant laboratory examinations in order to assess the patients' condition and guide treatments (Table 3 ). The results indicated that $23.7 \%$ of the patients (119/502) had leukopenia, which was more frequently seen in severe patients $(P<0.001)$. In patients with lymphocyte count $<1.0 \times 10^{9} / \mathrm{L}, 130 / 169(76.9 \%)$ patients eventually developed severe disease. The levels of other inflammatory 
Table 2 Clinical characteristics, radiographic, and etiology of patients with COVID-19

\begin{tabular}{|c|c|c|c|c|}
\hline Variables & All Patients $(n=550)$ & Severe $(n=178)$ & Non-severe $(n=372)$ & $p$ value \\
\hline Surgical history, n (\%) & $90(16.4 \%)$ & $24(13.5 \%)$ & $66(17.7 \%)$ & 0.207 \\
\hline \multicolumn{5}{|l|}{ Signs and symptoms, n (\%) } \\
\hline \multicolumn{5}{|l|}{ Fever } \\
\hline$<37.3^{\circ} \mathrm{C}$ & $178(32.3 \%)$ & $21(11.8 \%)$ & $157(42.3 \%)$ & $<0.001$ \\
\hline $37.3-38.0^{\circ} \mathrm{C}$ & $117(21.3 \%)$ & $35(19.7 \%)$ & $82(22.0 \%)$ & $<0.001$ \\
\hline $38.0-39.0^{\circ} \mathrm{C}$ & $204(37.1 \%)$ & $93(52.2 \%)$ & $111(29.8 \%)$ & $<0.001$ \\
\hline$>39.0^{\circ} \mathrm{C}$ & $51(9.3 \%)$ & $29(16.3 \%)$ & $22(5.9 \%)$ & $<0.001$ \\
\hline Dry cough & $257(46.7 \%)$ & $90(50.6 \%)$ & $167(44.9 \%)$ & 0.212 \\
\hline Dyspnea & $237(43.1 \%)$ & $119(66.9 \%)$ & $118(31.7 \%)$ & $<0.001$ \\
\hline Fatigue & $224(40.7 \%)$ & $91(51.1 \%)$ & $133(35.8 \%)$ & 0.001 \\
\hline Sputum production & $169(30.7 \%)$ & $70(39.3 \%)$ & 99 (26.6\%) & 0.002 \\
\hline Chill & $123(22.4 \%)$ & $52(29.2 \%)$ & $71(19.1 \%)$ & 0.008 \\
\hline Stomachache/Diarrhea & 75 (13.6\%) & $29(16.3 \%)$ & $46(12.4 \%)$ & 0.209 \\
\hline Nausea/Nomit & $70(12.7 \%)$ & $22(12.4 \%)$ & $48(12.9 \%)$ & 0.858 \\
\hline Myalgia & $57(10.4 \%)$ & $18(10.1 \%)$ & 39 (10.5\%) & 0.894 \\
\hline Sore throat & $46(8.8 \%)$ & $12(6.7 \%)$ & $34(9.1 \%)$ & 0.342 \\
\hline Tachycardia & $33(6.0 \%)$ & $17(9.6 \%)$ & $16(4.3 \%)$ & 0.015 \\
\hline Headache & $26(4.7 \%)$ & 7 (3.9\%) & $19(5.1 \%)$ & 0.544 \\
\hline Dizziness & $18(3.3 \%)$ & $4(2.2 \%)$ & $14(3.8 \%)$ & 0.350 \\
\hline Sneeze & $1(0.2 \%)$ & $0(0.0 \%)$ & $1(0.3 \%)$ & 1.000 \\
\hline Arthralgia & $1(0.2 \%)$ & $0(0.0 \%)$ & $1(0.3 \%)$ & 1.000 \\
\hline Multiple symptoms & $393(71.5 \%)$ & $162(91.0 \%)$ & $231(62.1 \%)$ & $<0.001$ \\
\hline Fever, cough ${ }^{a}$, and dyspnea & $130(23.6 \%)$ & $76(42.7 \%)$ & $54(14.5 \%)$ & $<0.001$ \\
\hline \multicolumn{5}{|l|}{ Radiographic findings ${ }^{\mathrm{b}}, \mathrm{n}(\%)$} \\
\hline Bilateral pneumonia & $378 / 482(78.4 \%)$ & 146/161 (90.7\%) & 232/321 (72.3\%) & $<0.001$ \\
\hline Unilateral pneumonia & $50 / 482(10.4 \%)$ & $5 / 161(3.1 \%)$ & $45 / 321(14.0 \%)$ & $<0.001$ \\
\hline Normal & $7 / 482(1.5 \%)$ & 0/161 (0\%) & $7 / 321(2.2 \%)$ & $<0.001$ \\
\hline Others & 47/482 (9.7\%) & 10/161 (6.2\%) & $37 / 321(11.5 \%)$ & $<0.001$ \\
\hline \multicolumn{5}{|l|}{ Etiological findings, n (\%) } \\
\hline \multicolumn{5}{|l|}{ Phlegm smear } \\
\hline Gram-positive bacilli & 3/34 (8.8\%) & $1 / 11(9.1 \%)$ & $2 / 23(8.7 \%)$ & 0.374 \\
\hline Gram-negative bacilli & 12/34 (35.4\%) & $3 / 11(27.3 \%)$ & 9/23 (39.1\%) & 0.374 \\
\hline Cocci & 10/34 (29.4\%) & 2/11 (18.2\%) & $8 / 23(34.8 \%)$ & 0.374 \\
\hline Fungus & $1 / 34(2.9 \%)$ & $1 / 11(9.1 \%)$ & $0 / 23(0.0 \%)$ & 0.374 \\
\hline Normal & $8 / 34(23.5 \%)$ & 4/11 (36.3\%) & $4 / 23(17.4)$ & 0.374 \\
\hline \multicolumn{5}{|c|}{ Mycoplasma/Chlamydia Pneumoniae antibody (IgM) } \\
\hline Positive & $27 / 350(7.7 \%)$ & $4 / 121(3.3 \%)$ & $23 / 229(10.0 \%)$ & 0.042 \\
\hline Negative & $323 / 350(92.3 \%)$ & $117 / 121(96.7 \%)$ & $206 / 229(90.0 \%)$ & 0.042 \\
\hline \multicolumn{5}{|l|}{ Respiratory pathogen antibody } \\
\hline Positive & 29/342 (8.5\%) & $13 / 123(10.6 \%)$ & $16 / 219(7.3 \%)$ & 0.299 \\
\hline Negative & $313 / 342(91.5 \%)$ & $110 / 123(89.4 \%)$ & 203/219 (92.7\%) & 0.299 \\
\hline \multicolumn{5}{|l|}{ CMV/EBV } \\
\hline Positive & 10/154 (6.5\%) & $4 / 54(7.4 \%)$ & $6 / 100(6.0 \%)$ & 1.000 \\
\hline Negative & 144/154 (93.5\%) & $50 / 54(92.6 \%)$ & $94 / 100(94.0 \%)$ & 1.000 \\
\hline
\end{tabular}


Table 2 Clinical characteristics, radiographic, and etiology of patients with COVID-19 (Continued)

\begin{tabular}{|c|c|c|c|c|}
\hline Variables & All Patients $(n=550)$ & Severe $(n=178)$ & Non-severe $(n=372)$ & $p$ value \\
\hline \multicolumn{5}{|l|}{ Influenza Virus Antigen } \\
\hline Positive & $3 / 265(1.1 \%)$ & 1/103 (1.0\%) & $2 / 162(1.2 \%)$ & 1.000 \\
\hline Negative & 262/265 (98.9\%) & 102/103 (99.0\%) & 160/162 (98.8\%) & 1.000 \\
\hline Admission & $9(6-14)$ & $10(7-12)$ & $9(5.75-15)$ & 0.797 \\
\hline Dyspnea & $0(0-7)$ & $2(0-8)$ & $0(0-6)$ & 0.007 \\
\hline Mechanical ventilation & $10(6-15)$ & $10(6.75-15)$ & - & - \\
\hline ARDS & $10(6-15)$ & $10(6.75-15)$ & - & - \\
\hline
\end{tabular}

Data are shown as median (IQR) or $\mathrm{n}(\%)$. ARDS Acute respiratory distress syndrome, CMV Cytomegalovirus. EBV Epstein-Barr virus

${ }^{a}$ Cough includes dry cough and expectoration

${ }^{b}$ Radiographic findings include the findings of both the chest X-ray and lung CT scan. When "viral pneumonia" was reported only, without a description of the lesion sites, the results were marked by others

indicators such as procalcitonin (PCT), highly-sensitive C-reactive protein (hsCRP), and erythrocyte sedimentation rate (ESR) were increased in severe patients compared to non-severe patients $(P<0.001)$. In addition, myocardial enzymes were elevated in severe patients, and $85 / 128(66.4 \%)$ of severe patients presented elevated NT-proBNP levels $(P<0.001)$. Elevation of alanine and aspartate aminotransferase occurred more frequently in severe patients, and 163/175 (93.0\%) severe patients had hypoproteinemia $(P<0.001)$. A relatively small number of patients developed a reduced glomerular filtration rate, but it was more commonly seen in severe patients (24/176, 13.6\%, $P<0.001)$. Furthermore, more patients in the severe group $(108 / 133,81.2 \%)$ had elevated Ddimer levels compared to non-severe patients. Moreover, severe patients were more likely to be associated with electrolyte disorders. Blood gas analysis revealed that $75.2 \%(124 / 165)$ of severe patients had an oxygen index (OI) $<300$ at admission, of which 26 patients had OI < 100. There was no difference in the proportion of patients with hyperlactatemia between the two groups $(P=$ 0.172).

Lymphocyte count, troponin $\mathrm{T}$, serum creatinine, $\mathrm{D}$ dimer level, and OI were closely monitored and compared between the severe and non-severe groups (Fig. 1). The lymphocyte counts were lower in severe patients but increased more robustly after day 7 compared with non-severe patients. Troponin $\mathrm{T}$ and $\mathrm{D}$-dimer levels were higher in severe patients and peaked around the 4th day after admission. There was no significant difference in creatinine levels between the two groups except on 1st day of admission. In addition, severe hypoxemia was more common in severe patients.

In this cohort, only a very small number of patients were co-infected with other pathogens such as bacteria, influenza virus, and atypical pathogens (Table 2). Among all patients, 482 patients (87.6\%) had completed chest radiographs or lung CT scans during hospitalization. For all 161 patients that progressed into advanced stages with radiologic assessments, 146 (90.7\%) had bilateral lung lesions. Only $72.3 \%$ (232/321) of the non-severe patients developed bilateral lung lesions (Table 2).

\section{Complications}

The most common complications were acute myocardial injury $(n=111,20.2 \%)$, secondary infection $(n=110$, $20.0 \%)$, ARDS $(n=69,12.5 \%)$, acute renal injury $(n=45$, $8.2 \%)$, shock $(n=40,7.3 \%)$, and disseminated intravascular coagulation (DIC) $(n=20,3.6 \%)$. Unsurprisingly, severe patients were more likely to develop complications (Table 4).

\section{Identification of risk factors for severe cases}

The multivariable logistic regression analysis showed that age $\geq 60$ years $(\mathrm{OR}=3.02,95 \% \mathrm{CI}: 1.13-8.08, P=$ $0.028)$ and $\mathrm{D}$-dimer $>0.243 \mu \mathrm{g} / \mathrm{ml} \quad(\mathrm{OR}=2.73,95 \% \mathrm{CI}$ : $1.01-7.39, P=0.047)$ were independently associated with severe cases (Table 5). A decrease in OI $(\mathrm{OR}=0.984$, 95\%CI: $0.980-0.999, P<0.001)$ was also independently associated with disease deterioration.

\section{Treatments}

All patients (100.0\%) were given intermittent or continuous oxygen inhalation therapy to improve the clinical symptoms (Table 4). Among the severe patients, 20.2\% required invasive ventilator support, $18.0 \%$ required non-invasive ventilator, and $2.8 \%$ required high-flow nasal cannula, while the remaining patients were treated with nasal catheters/masks for oxygen therapy. No ECMO was used.

Among the patients, $79.1 \%$ were treated with antibiotics, and $231(42.0 \%)$ were treated with more than one type of antibiotics. The choice of antibiotics was based on the local epidemiological situation in Wuhan. Most of the patients with severe diseases were treated with a combination of moxifloxacin and cefoperazone /sulbactam. The critical patients are often treated with imipenem or biapenem. Non-critical patients often received moxifloxacin, combined cefoperazone/sulbactam, or azithromycin. The most frequently used drugs were 
Table 3 Laboratory results of patients with COVID-19 on hospital admission

\begin{tabular}{|c|c|c|c|c|}
\hline Variables & All Patients $(n=550)$ & Severe $(n=178)$ & Non-severe $(n=372)$ & $p$ value \\
\hline \multicolumn{5}{|c|}{ Blood tests, n/total n (\%) } \\
\hline \multicolumn{5}{|c|}{ Leucocytes $\left(\times 10^{9} / \mathrm{L}\right)$} \\
\hline$<4$ & 119/502(23.7\%) & 21/169(12.4\%) & 98/333(29.4\%) & $<0.001$ \\
\hline $4-10$ & $335 / 502(66.7 \%)$ & $110 / 169(65.1 \%)$ & 225/333(67.6\%) & $<0.001$ \\
\hline$>10$ & 48/502(9.6\%) & $38 / 169(22.5 \%)$ & 10/333(3.0\%) & $<0.001$ \\
\hline \multicolumn{5}{|c|}{ Neutrophil percentage (\%) } \\
\hline $40-75$ & $321 / 502(63.9 \%)$ & $47 / 169(27.8 \%)$ & 274/333(82.3\%) & $<0.001$ \\
\hline$>75$ & $175 / 502(34.9 \%)$ & $122 / 169(72.2 \%)$ & $53 / 333(15.9 \%)$ & $<0.001$ \\
\hline \multicolumn{5}{|c|}{ Lymphocyte percentage (\%) } \\
\hline$<20$ & 230/502(45.8\%) & 143/169(84.6\%) & 87/333(26.1\%) & $<0.001$ \\
\hline $20-50$ & $267 / 502(53.2 \%)$ & 26/169(15.4\%) & $241 / 333(72.4 \%)$ & $<0.001$ \\
\hline \multicolumn{5}{|c|}{ Lymphocytes (×109/L) } \\
\hline$<1.0$ & 250/502(49.8\%) & 130/169(76.9\%) & $120 / 333(36.0 \%)$ & $<0.001$ \\
\hline$\geq 1.0$ & $252 / 502(50.2 \%)$ & $39 / 169(23.1 \%)$ & 213/333(64.0\%) & $<0.001$ \\
\hline \multicolumn{5}{|c|}{ Hemoglobin $(\mathrm{g} / \mathrm{L})$} \\
\hline Normal & $317 / 502(63.1 \%)$ & 108/169(63.9\%) & 209/333(62.8\%) & 0.802 \\
\hline Decreased & 185/502(36.9\%) & 61/169(36.1\%) & $124 / 333(37.2 \%)$ & 0.802 \\
\hline \multicolumn{5}{|c|}{ Platelets $\left(\times 10^{9} / \mathrm{L}\right)$} \\
\hline$<100$ & $34 / 502(6.8 \%)$ & 13/169(7.7\%) & $21 / 333(6.3 \%)$ & 0.559 \\
\hline$\geq 100$ & $468 / 502(93.2 \%)$ & 156/169(92.3\%) & 312/333(93.7\%) & 0.559 \\
\hline \multicolumn{5}{|c|}{ Inflammatory parameters-no./total no. (\%) } \\
\hline \multicolumn{5}{|c|}{ Procalcitonin (ng/ml) } \\
\hline$\leq 0.1$ & 279/393(71.0\%) & $63 / 149(42.3 \%)$ & 216/244(88.5\%) & $<0.001$ \\
\hline$>0.1$ & $117 / 393(29.0 \%)$ & $86 / 149(57.7 \%)$ & 28/244(11.5\%) & $<0.001$ \\
\hline \multicolumn{5}{|l|}{ hsCRP (mg/L) } \\
\hline$\leq 3$ & $120 / 404(29.7 \%)$ & $5 / 136(3.7 \%)$ & $115 / 268(42.9 \%)$ & $<0.001$ \\
\hline$>3$ & $284 / 404(70.3 \%)$ & 131/136(96.3\%) & $153 / 268(57.1 \%)$ & $<0.001$ \\
\hline \multicolumn{5}{|l|}{$\operatorname{ESR}(\mathrm{mm} / \mathrm{h})$} \\
\hline$\leq 15$ & $74 / 185(40.0 \%)$ & $3 / 55(5.5 \%)$ & $71 / 130(54.6 \%)$ & $<0.001$ \\
\hline$>15$ & $111 / 185(60.0 \%)$ & $52 / 55(94.5 \%)$ & $59 / 130(45.4 \%)$ & $<0.001$ \\
\hline \multicolumn{5}{|c|}{ Myocardial enzyme-no./total no. (\%) } \\
\hline \multicolumn{5}{|c|}{ CK-MB (ng/mL) } \\
\hline$\leq 6.22$ & 399/422(94.5\%) & 130/146(89.0\%) & $269 / 276(97.5 \%)$ & $<0.001$ \\
\hline$>6.22$ & 23/422(5.5\%) & 16/146(11.0\%) & $7 / 276(2.5 \%)$ & $<0.001$ \\
\hline \multicolumn{5}{|c|}{ Troponin T (ng/ml) } \\
\hline$\leq 0.014$ & $331 / 438(75.6 \%)$ & $82 / 161(50.9 \%)$ & 249/277(89.9\%) & $<0.001$ \\
\hline$>0.014$ & 107/438(24.4\%) & 79/161(49.1\%) & $28 / 277(10.1 \%)$ & $<0.001$ \\
\hline \multicolumn{5}{|c|}{ Heart Failure Indicator-no./total no. (\%) } \\
\hline \multicolumn{5}{|l|}{$\mathrm{BNP}(\mathrm{pg} / \mathrm{ml})$} \\
\hline$\leq 222$ & 166/291(57.0\%) & 43/128(33.6\%) & $123 / 163(75.5 \%)$ & $<0.001$ \\
\hline$>222$ & $125 / 291(43.0 \%)$ & $85 / 128(66.4 \%)$ & $40 / 163(24.5 \%)$ & $<0.001$ \\
\hline \multicolumn{5}{|c|}{ Liver function-no./total no. (\%) } \\
\hline \multicolumn{5}{|c|}{ Alanine transaminase (IU/L) } \\
\hline$\leq 50$ & $443 / 511(86.7 \%)$ & 136/175(77.7\%) & $307 / 336(91.4 \%)$ & $<0.001$ \\
\hline
\end{tabular}


Table 3 Laboratory results of patients with COVID-19 on hospital admission (Continued)

\begin{tabular}{|c|c|c|c|c|}
\hline Variables & All Patients $(n=550)$ & Severe $(n=178)$ & Non-severe $(n=372)$ & $p$ value \\
\hline$>50$ & 68/511(13.3\%) & $39 / 175(22.3 \%)$ & 29/336(8.6\%) & $<0.001$ \\
\hline \multicolumn{5}{|c|}{ Aspartate aminotransferase (IU/L) } \\
\hline$\leq 40$ & 393/511(76.9\%) & $95 / 175(54.3 \%)$ & 298/336(88.7\%) & $<0.001$ \\
\hline$>40$ & $118 / 511(23.1 \%)$ & $80 / 175(45.7 \%)$ & 38/336(11.3\%) & $<0.001$ \\
\hline \multicolumn{5}{|c|}{ Albumin (g/L) } \\
\hline$<40$ & $345 / 511(67.5 \%)$ & 163/175(93.1\%) & $182 / 336(54.2 \%)$ & $<0.001$ \\
\hline $40-55$ & $166 / 511(32.5 \%)$ & $12 / 175(6.9 \%)$ & $154 / 336(45.8 \%)$ & $<0.001$ \\
\hline \multicolumn{5}{|c|}{ Coagulation Function, n/total n (\%) } \\
\hline \multicolumn{5}{|l|}{ APTT (S) } \\
\hline $24.6-35.4$ & $364 / 430(84.7 \%)$ & 136/158(86.1\%) & 228/272(83.8\%) & 0.532 \\
\hline$>35.4$ & $66 / 430(15.3 \%)$ & 22/158(13.9\%) & $44 / 272(16.2 \%)$ & 0.532 \\
\hline \multicolumn{5}{|c|}{ D-dimer $(\mu \mathrm{g} / \mathrm{ml})$} \\
\hline$\leq 0.243$ & $182 / 364(50.0 \%)$ & 25/133(18.8\%) & $157 / 231(68.0 \%)$ & $<0.001$ \\
\hline$>0.243$ & $182 / 364(50.0 \%)$ & 108/133(81.2\%) & $74 / 231(32.0 \%)$ & $<0.001$ \\
\hline \multicolumn{5}{|c|}{ Electrolyte, n/total n (\%) } \\
\hline \multicolumn{5}{|c|}{ Potassium (mmol/L) } \\
\hline$>5.3$ & 39/504(7.7\%) & $22 / 172(12.8 \%)$ & $17 / 332(5.1 \%)$ & $<0.001$ \\
\hline $3.5-5.3$ & $389 / 504(77.2 \%)$ & 109/172(63.4\%) & 280/332(84.4\%) & $<0.001$ \\
\hline$<3.5$ & 76/504(15.1\%) & $41 / 172(23.8 \%)$ & $35 / 332(10.5 \%)$ & $<0.001$ \\
\hline \multicolumn{5}{|c|}{ Sodium (mmol/L) } \\
\hline$<137$ & 72/504(14.3\%) & $44 / 172(25.6 \%)$ & 28/332(8.4\%) & $<0.001$ \\
\hline $137-147$ & $411 / 504(81.5 \%)$ & $115 / 172(66.8 \%)$ & 296/332(89.2\%) & $<0.001$ \\
\hline$>147$ & $21 / 504(4.2 \%)$ & 13/172(7.6\%) & $8 / 332(2.4 \%)$ & $<0.001$ \\
\hline \multicolumn{5}{|c|}{ Renal Function, n/total n (\%) } \\
\hline \multicolumn{5}{|c|}{ Creatinine $(\mu \mathrm{mol} / \mathrm{L})$} \\
\hline$\leq 111$ & $480 / 508(94.5 \%)$ & 160/176(90.9\%) & $320 / 332(96.4 \%)$ & 0.010 \\
\hline$>111$ & 28/508(5.5\%) & 16/176(9.1\%) & $12 / 332(3.6 \%)$ & 0.010 \\
\hline \multicolumn{5}{|l|}{ GFR } \\
\hline$<66$ & $36 / 508(7.1 \%)$ & 24/176(13.6\%) & $12 / 332(3.6 \%)$ & $<0.001$ \\
\hline$\geq 66$ & $472 / 508(92.9 \%)$ & $152 / 176(86.4 \%)$ & $320 / 332(96.4 \%)$ & $<0.001$ \\
\hline \multicolumn{5}{|c|}{ Arterial blood gas analysis, n/total n (\%) } \\
\hline \multicolumn{5}{|l|}{$\mathrm{PH}$} \\
\hline$<7.35$ & 23/338(6.8\%) & $14 / 165(8.5 \%)$ & 9/173(5.2\%) & $<0.001$ \\
\hline $7.35-7.45$ & 233/338(68.9\%) & $90 / 165(54.5 \%)$ & 143/173(82.7\%) & $<0.001$ \\
\hline$>7.45$ & $82 / 338(24.3 \%)$ & $61 / 165(37.0 \%)$ & 21/173(12.1\%) & $<0.001$ \\
\hline \multicolumn{5}{|c|}{ Oxygenation index } \\
\hline$<100$ & 26/338(7.7\%) & 26/165(15.8\%) & 0/173(0.0\%) & $<0.001$ \\
\hline $100-300$ & 98/338(29.0\%) & 98/165(59.4\%) & $0 / 173(0.0 \%)$ & $<0.001$ \\
\hline$>300$ & 214/338(63.3\%) & $41 / 165(24.8 \%)$ & 173/173(100.0\%) & $<0.001$ \\
\hline \multicolumn{5}{|c|}{ PCO2 (mmHg) } \\
\hline$<35$ & 73/338(21.6\%) & $52 / 165(31.5 \%)$ & 21/173(12.1\%) & $<0.001$ \\
\hline $35-45$ & 185/338(54.7\%) & $90 / 165(54.6 \%)$ & 95/173(55.0\%) & $<0.001$ \\
\hline$>45$ & 80/338(23.7\%) & 23/165(13.9\%) & 57/173(32.9\%) & $<0.001$ \\
\hline
\end{tabular}

Lactic acid (mmol/L) 
Table 3 Laboratory results of patients with COVID-19 on hospital admission (Continued)

\begin{tabular}{rllll}
\hline Variables & All Patients $(\boldsymbol{n}=\mathbf{5 5 0})$ & Severe $(\boldsymbol{n}=\mathbf{1 7 8})$ & Non-severe $(\boldsymbol{n}=\mathbf{3 7 2})$ & $\boldsymbol{p}$ value \\
\hline$\leq 2.2$ & $245 / 338(72.5 \%)$ & $114 / 165(69.1 \%)$ & $131 / 173(75.7 \%)$ & 0.172 \\
$>2.2$ & $93 / 338(27.5 \%)$ & $51 / 165(30.9 \%)$ & $42 / 173(24.3 \%)$ & 0.172
\end{tabular}

The data were expressed in the form of $\mathrm{n} / \mathrm{N}(\%)$, where $\mathrm{N}$ represents the total number of patients with available data

hsCRP Hypersensitive c-reactive protein, ESR Erythrocyte sedimentation rate, CKMB Creatine kinase isoenzyme, BNP B-type natriuretic peptide, APTT Activated partial thromboplastin time, GFR Glomerular filtration rate, PCO2 Partial pressure of carbon dioxide

moxifloxacin $(n=407,74.0 \%)$, cephalosporins/ sulbactam $(n=186,33.8 \%)$, carbapenems $(n=61,11.1 \%)$, and azithromycin $(n=52,9.5 \%)$. A higher percentage of patients in the severe group received intravenous or oral glucocorticoids compared with the non-severe patients (122/178, $68.5 \%$ vs. $69 / 372,18.5 \%, P<0.001)$.

Among all patients, $81.6 \%$ were treated with antiviral drugs, and the remaining $18.4 \%$ were treated only with traditional Chinese medicine. The outcomes of the patients treated with antiviral drugs are shown in Table 6. The antiviral drugs used in this study were arbidol $(n=$ $240,43.6 \%)$, oseltamivir $(n=216,39.3 \%)$, ribavirin $(n=$ 152, 27.6\%), lopinavir/ritonavir $(n=21,3.8 \%)$, and $\alpha-$ interferon $(n=20,3.6 \%)$. Arbidol was more effective than ribavirin $(73.3 \%$ vs. $41.2 \%, P=0.029)$ in treating severe patients as single-drug therapy when considering symptom relief, clinical outcome, and discharge rate. Similarly, in severe patients who were treated with two drugs, arbidol combined with ribavirin or oseltamivir also had better efficacy. There were no significant differences identified among the other treatments. Some patients also received immunotherapies, including human immunoglobulin infusion $(n=52,9.5 \%)$ and thymosin $(n=10,1.8 \%)$. Vasoactive drugs were used in 34 severe cases, and continuous blood purification therapy was used in two cases.

\section{Patient outcomes}

After treatment, 474 (86.1\%) patients' conditions were improved, $24(4.4 \%)$ patients were transferred to superior hospitals, and $52(9.5 \%)$ patients passed away due to multiple organ failure (63.5\%), respiratory failure $(30.8 \%)$, circulatory failure $(3.8 \%)$, and septic shock (1.9\%). Next, the MuLBSTA scoring system was used to score the mortality cases and showed that 46 patients belonged to the high-risk group of death, with a median score of 17 (15-17), while six cases were in the low-risk group, with a median score of $9(8.25-10.5)$. The median hospitalization time was 16 (IQR, 9-26) days for all patients and 22 (IQR, 13-30) days for severe patients.

\section{Discussion}

Because the incubation period of COVID-19 can be up to 3 weeks [1-4], the early identification of patients at higher risk of severe disease is important to implement timely medical strategies. This could save time and energy in the context of the exhausted healthcare systems. The results suggest that age $\geq 60$ years, D-dimer $>$ $0.243 \mu \mathrm{g} / \mathrm{ml}$, and lower oxygenation index were associated with severe COVID-19. Therefore, the patients presenting those characteristics could be more aggressively managed from the start in order to prevent complications. In addition, arbidol might provide more clinical benefits in treating patients with severe COVID-19 compared with other antiviral drugs.

A total of 550 patients diagnosed with COVID-19 were included in this study. Inconsistent with the literature, there were more females $(53.1 \%)$ than males in the present study [1-4], but there were more male patients among severe cases. This discrepancy can be due to many factors, including the transmission route, willingness to undergo screening, and socioeconomic factors. Epidemiology tracing identified 170 (30.9\%) of patients with 2019-nCoV having a history of contact with an infected individual and 38 (6.9\%) due to a recent visit to a COVID-19-designated hospital. The remaining of the patients had no clear source of infection. Those results highlight the need for refraining from having contacts and from enforcing physical distancing, from avoiding visiting hospitals known to treat COVID-19 and visiting other hospitals, and that many patients might have been infected through asymptomatic, either because those patients were asymptomatic carriers or because symptom onset did not occur yet. This will have to be examined in future studies.

In this study, $42.3 \%$ of the non-severe patients did not have a fever at diagnosis, which was lower than what was reported by Guan et al. [4]. Upon hospital admission, $42.7 \%$ of the patients who eventually progressed to severe COVID-19 had the typical triple signs (fever, cough, and dyspnea), while the triple signs were observed in only $14.5 \%$ of the non-severe patients. Unsurprisingly, severe patients often had more comorbidities. Hypertension, diabetes, cardiovascular diseases, and malignancy were the most common underlying diseases observed in patients with severe COVID-19. Older age was also observed to be associated with severe COVID-19, which is consistent with recent studies $[4,28]$, but whether this is because older patients can be frailer and weaker or because older individuals often have more comorbidities is still unknown. 


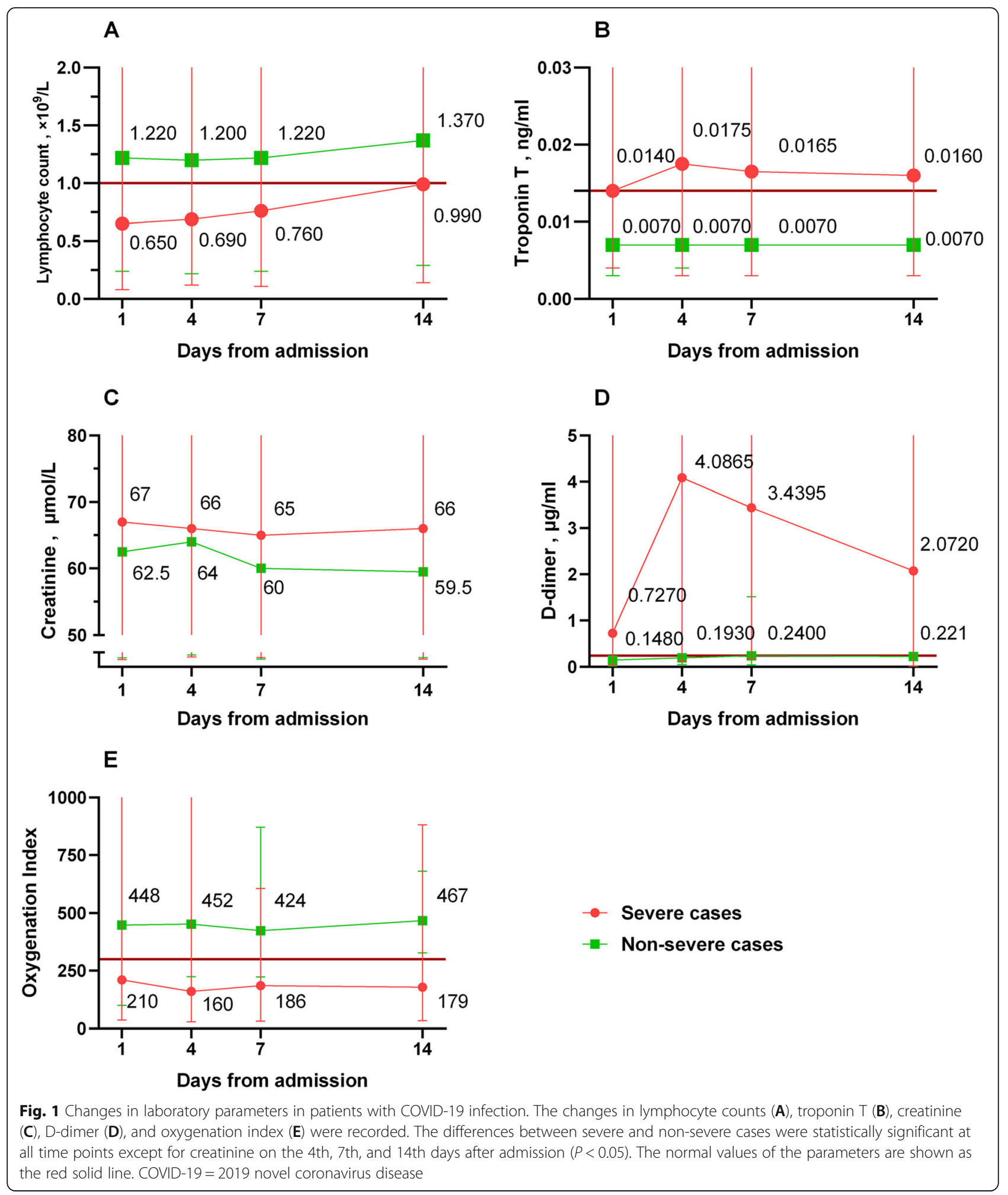

As for the laboratory tests, $66.7 \%$ of the patients in the study had normal leukocyte count, and a quarter of the patients had decreased WBC counts. For $9.6 \%$ of patients who had an elevated WBC, secondary infections were often the cause of the elevated WBC. In addition,
$49.8 \%$ of the patients presented with decreased lymphocyte counts, of which $52.0 \%$ (130/250) were severe cases. The incidence of anemia and thrombocytopenia was 36.9 and $6.8 \%$, respectively, without differences according to disease severity. Cardiac enzymes, troponin $\mathrm{T}$, 
Table 4 Complications, treatment, and prognosis of patients with COVID-19

\begin{tabular}{|c|c|c|c|c|}
\hline Variables & All Patients $(n=550)$ & Severe $(n=178)$ & Non-severe $(n=372)$ & $p$ value \\
\hline \multicolumn{5}{|l|}{ Complications n, \% } \\
\hline Acute myocardial injury & $111(20.2 \%)$ & $76(42.7 \%)$ & $35(9.4 \%)$ & $<0.001$ \\
\hline Secondary infection & $110(20.0 \%)$ & $82(46.1 \%)$ & $28(7.5 \%)$ & $<0.001$ \\
\hline ARDS & $69(12.5 \%)$ & $69(38.8 \%)$ & 0 & $<0.001$ \\
\hline Acute kidney injury & $45(8.2 \%)$ & $33(18.5 \%)$ & $12(3.2 \%)$ & $<0.001$ \\
\hline Shock & $40(7.3 \%)$ & $39(21.9 \%)$ & $1(0.3 \%)$ & $<0.001$ \\
\hline $\mathrm{DIC}$ & $20(3.6 \%)$ & $11(6.2 \%)$ & $9(2.4 \%)$ & 0.028 \\
\hline \multicolumn{5}{|l|}{ Treatment n.\% } \\
\hline Antiviral therapy & $449(81.6 \%)$ & 162(91.0\%) & $287(77.2 \%)$ & $<0.001$ \\
\hline \multicolumn{5}{|l|}{ Antibacterial therapy } \\
\hline one kind & $204(37.1 \%)$ & $40(22.5 \%)$ & $164(44.1 \%)$ & $<0.001$ \\
\hline$\geq$ two kinds & $231(42.0 \%)$ & $136(76.4 \%)$ & $95(25.5 \%)$ & $<0.001$ \\
\hline Antifungal therapy & $10(1.8 \%)$ & $8(4.5 \%)$ & $2(0.5 \%)$ & 0.004 \\
\hline Glucocorticoids therapy & 191(34.7\%) & $122(68.5 \%)$ & $69(18.5 \%)$ & $<0.001$ \\
\hline \multicolumn{5}{|l|}{ Immunotherapy } \\
\hline Human immunoglobulin & $52(9.5 \%)$ & $23(12.9 \%)$ & $29(7.8 \%)$ & 0.055 \\
\hline Thymosin & $10(1.8 \%)$ & $6(3.4 \%)$ & $4(1.1 \%)$ & 0.123 \\
\hline Vasoactive drug & $34(6.2 \%)$ & $34(19.1 \%)$ & 0 & $<0.001$ \\
\hline CRRT & $2(0.4 \%)$ & $2(1.1 \%)$ & 0 & 0.104 \\
\hline \multicolumn{5}{|l|}{ Respiratory support-no.\% } \\
\hline Nasal catheter/Mask oxygen & $477(86.8 \%)$ & $105(59.0 \%)$ & $372(100.0 \%)$ & $<0.001$ \\
\hline High-flow nasal cannula & $5(0.9 \%)$ & $5(2.8 \%)$ & 0 & $<0.001$ \\
\hline Noninvasive ventilation & $32(5.8 \%)$ & $32(18.0 \%)$ & 0 & $<0.001$ \\
\hline Invasive ventilation & $36(6.5 \%)$ & $36(20.2 \%)$ & 0 & $<0.001$ \\
\hline ECMO & 0 & 0 & 0 & - \\
\hline \multicolumn{5}{|l|}{ prognosis-no.\% } \\
\hline Transfer & $24(4.4 \%)$ & $16(9.0 \%)$ & $8(2.2 \%)$ & $<0.001$ \\
\hline Improved & $474(86.1 \%)$ & $110(61.8 \%)$ & $364(97.8 \%)$ & $<0.001$ \\
\hline Death & $52(9.5 \%)$ & $52(29.2 \%)$ & 0 & $<0.001$ \\
\hline Multiple system and organ failure & $33 / 52(63.5 \%)$ & $33 / 52(63.5 \%)$ & - & - \\
\hline Respiratory failure & 16/52 (30.8\%) & 16/52 (30.8\%) & - & - \\
\hline Circulatory failure & $2 / 52(3.8 \%)$ & $2 / 52(3.8 \%)$ & - & - \\
\hline Septic shock & $1 / 52(1.9 \%)$ & $1 / 52(1.9 \%)$ & - & - \\
\hline Negative conversion time of RNA Detection, Median (IQR)-days & $10(6-16)$ & $13(8-18)$ & $9(6-16)$ & 0.016 \\
\hline Length of hospital stay, Median (IQR)-days & $16(9-26)$ & $22(13-30)$ & $15(9-22.75)$ & $<0.001$ \\
\hline
\end{tabular}

transaminase, creatinine, and other organ injury indicators were also increased to varying degrees in some patients. Both hsCRP and ESR were increased in most patients, especially in severe patients. This highlights the systemic nature of the disease and that the patients should be comprehensively assessed. The increased inflammatory indicators suggested that SARS-CoV-2 tips the balance of the immune system towards a cytokine storm that contributes to patient deterioration and mortality, as observed in various infections $[29,30]$. Recent biopsy reports by $\mathrm{Xu}$ et al. [31] also indicated an increase of proinflammatory CCR4+ CCR6+ Th17 cells in the peripheral blood that might lead to systemic inflammatory responses and contribute to diffuse alveolar injury and pulmonary hyaline membrane formation. That evidence suggests that the systemic inflammatory response is an important factor leading to poor COVID-19 prognosis, as supported by the literature [29, 30]. Unfortunately, due to the limited conditions of the hospital, no cytokine or other related testing was performed in 
Table 5 Early warning indicators for the occurrence of severe cases with COVID-19

\begin{tabular}{|c|c|c|c|c|}
\hline & Univariable OR $(95 \% \mathrm{Cl})$ & $p$ value & Multivariable OR $(95 \% \mathrm{Cl})$ & $p$ value \\
\hline \multicolumn{5}{|l|}{ Demographics and clinical characteristics } \\
\hline \multicolumn{5}{|l|}{ Age, years } \\
\hline$\geq 60$ & $3.985(2.701-5.879)$ & $<0.001$ & $3.022(1.130-8.083)$ & 0.028 \\
\hline \multicolumn{5}{|l|}{ Sex } \\
\hline Male & $2.034(1.415-2.924)$ & $<0.001$ & & \\
\hline \multicolumn{5}{|l|}{ Comorbidity } \\
\hline Hypertension & $2.152(1.484-3.121)$ & $<0.001$ & $0.724(0.263-1.999)$ & 0.531 \\
\hline Diabetes & $2.178(1.336-3.550)$ & 0.002 & & \\
\hline Heart disease & $2.023(1.152-3.552)$ & 0.014 & & \\
\hline Cancer & $2.155(0.88-5.276)$ & 0.093 & & \\
\hline \multicolumn{5}{|l|}{ Temperature, ${ }^{\circ} \mathrm{C}$} \\
\hline$\geq 38.0$ & $2.010(1.103-3.663)$ & 0.023 & $1.355(0.536-3.423)$ & 0.521 \\
\hline \multicolumn{5}{|l|}{ Symptom } \\
\hline More than one sign or symptom & $2.841(1.941-4.157)$ & $<0.001$ & & \\
\hline Fever, cough and dyspnea & $2.373(1.580-3.566)$ & $<0.001$ & & \\
\hline \multicolumn{5}{|l|}{ Radiographic and laboratory findings } \\
\hline \multicolumn{5}{|l|}{ Radiographic findings ${ }^{\text {a }}$} \\
\hline Bilateral pneumonia & 6.419 (2.253-18.287) & $<0.001$ & & \\
\hline \multicolumn{5}{|l|}{ Leucocytes $\left(\times 10^{9} / \mathrm{L}\right)$} \\
\hline$<4$ & $0.119(0.053-0.267)$ & $<0.001$ & & \\
\hline$>10$ & $0.056(0.023-0.138)$ & $<0.001$ & & \\
\hline \multicolumn{5}{|l|}{ Lymphocyte count $\left(\times 10^{9} / \mathrm{L}\right)$} \\
\hline $\mathrm{LN}<1.0$ & $3.297(2.213-4.912)$ & $<0.001$ & $1.903(0.736-4.923)$ & 0.184 \\
\hline \multicolumn{5}{|l|}{ Procalcitonin (ng/ml) } \\
\hline$>0.1$ & $6.860(4.222-11.146)$ & $<0.001$ & & \\
\hline \multicolumn{5}{|l|}{ Troponin T (ng/ml) } \\
\hline$>0.014$ & 9.465 (5.412-16.554) & $<0.001$ & & \\
\hline \multicolumn{5}{|l|}{ D-dimer ( $\mu \mathrm{g} / \mathrm{ml})$} \\
\hline$>0.243$ & 4.375 (2.191-8.734) & $<0.001$ & $2.734(1.012-7.387)$ & 0.047 \\
\hline \multicolumn{5}{|l|}{ Glomerular filtration rate } \\
\hline$<66$ & 4.375 (2.191-8.734) & $<0.001$ & & \\
\hline Oxygenation index & 0.986 (0.983-0.989) & $<0.001$ & $0.984(0.980-0.989)$ & $<0.001$ \\
\hline \multicolumn{5}{|l|}{ Lactic acid (mmol/L) } \\
\hline$>2.2$ & $1.547(0.939-2.551)$ & 0.087 & & \\
\hline
\end{tabular}

Univariable and multivariable logistic regression analyses were performed, and six variables were selected for further multivariable. OR Odds ratio

${ }^{\text {a }}$ Radiographic findings include the findings of both chest X-ray and lung CT scan

this study. Future studies should aim to carefully examine the various cytokines involved in COVID-19 and in relation to disease severity.

It has been estimated that the mortality rate in severe cases was over 50\% [32]. Therefore, it is critical to identify patients with an increased risk of disease progression. In the present study, the multivariable logistic regression analysis showed that age $\geq 60$ years, D-dimer $>0.243 \mu \mathrm{g} / \mathrm{ml}$, and decreased OI might be risk factors for patient deterioration. The importance of aging in determining the COVID-19 prognosis was consistent with previous studies that aimed to identify prognosis factors for SARS or MERS infections [28, 33-35]. The coagulation dysfunction we observed in this study was consistent with previous studies $[1-3,5]$. Severe patients were more likely to develop coagulation and fibrinolysis disorders, especially the elevation of D-dimer levels. Similar to other severe viral pneumonia, the cause might be the 
Table 6 Antiviral efficacy in patients with COVID-19

\begin{tabular}{lllll}
\hline & \multicolumn{2}{l}{ Severe $(\boldsymbol{n}=\mathbf{1 7 8})$} & \multicolumn{2}{l}{ Non-severe $(\boldsymbol{n}=\mathbf{3 7 2})$} \\
\hline Ribavirin & $7 / 17$ & $41.2 \%$ & $22 / 23$ & $95.7 \%$ \\
Oseltamivir & $34 / 53$ & $64.2 \%$ & $49 / 49$ & $100.0 \%$ \\
Arbidol & $22 / 30$ & $73.3 \%$ & $109 / 110$ & $99.1 \%$ \\
Lopinavir/ritonavir & $0 / 0$ & - & $2 / 2$ & $100 \%$ \\
Ribavirin+Oseltamivir & $9 / 20$ & $45.0 \%$ & $38 / 38$ & $100 \%$ \\
Ribavirin+Arbidol & $12 / 12$ & $100 \%$ & $18 / 19$ & $94.7 \%$ \\
Arbidol+Oseltamivir & $15 / 17$ & $88.2 \%$ & $14 / 16$ & $87.5 \%$ \\
\hline
\end{tabular}

The data were expressed in the form of $n / N(\%)$, where $n$ represents the number of patients with clinical disease improvement, $\mathrm{N}$ represents the total number of patients receiving corresponding drugs

sepsis-induced inflammatory cytokine storm affecting multiple endogenous and exogenous coagulation pathways and fibrinolysis that ultimately lead to thrombosis formation [17, 29]. Therefore, special attention should be paid to severe patients with long-term bed rest, advanced age, and complicated underlying diseases, especially in the presence of coagulation abnormalities. Appropriate anticoagulant treatment might be considered in such patients in order to prevent the occurrence of deep vein thrombosis (DVT) and related complications [36-38]. In the present study, a reduction in OI was associated with increased mortality. Similarly, Liu et al. [39] found that the lung injury Murray score and OI could predict the prognosis of COVID-19. Therefore, early recognition of these three indicators upon hospital admission is critical, so appropriate medical strategies can be adjusted, and more importantly, the nearly exhausted medical support force can be redistributed. This is especially important because when the patients are admitted, the exact interval between infection and symptom onset is unknown, and the exact time until an eventual disease progression is also unknown.

In this study, 435 patients (79.1\%) received at least one antibiotic in the hospital, but only $110(20.0 \%)$ of them were confirmed with secondary bacterial infection (some cases were accompanied by fungal infection). Therefore, more attention should be paid to the indication of antibiotic use and avoid antibiotic overuse. Prophylaxis for eventual complicating secondary bacterial or fungal infections can be indicated in some cases, but additional studies are necessary to determine who they might be. Since patients with severe and critical COVID-19 have a compromised immune system $[40,41]$, the rationale for prophylactic antibiotics is to avoid a secondary infection that might worsen the condition of the patients and his prognosis. In addition, 122 severe patients received intravenous or oral glucocorticoid treatment. Among these patients, 79 had an improved condition, but 43 eventually died. Nevertheless, the rate of improvement was relatively high $(83.9 \%$ vs. $64.8 \%, P=0.009)$ in severe patients who did not receive glucocorticoids. It is important to point out that the patients who received glucocorticoids also had a higher rate of secondary infections compared with patients who did not receive glucocorticoids $(36.9 \%$ vs. $19.6 \%, P=0.021)$. This is consistent with several recent studies that suggested that glucocorticoids are not beneficial for patients with viral infections [42, 43], but contradicts recent findings that suggest that corticosteroids decrease the mortality of COVID-19, but the level of evidence is low [44]. A study in SARs showed that early glucocorticoids increased the viral load [45], and another study in MERS reported delayed virus clearance [46]. Even though this study was not powered to analyze the benefit and risks of using glucocorticoids in COVID-19, the data suggest that glucocorticoids failed to improve the prognosis and increased the risk of secondary infection.

Another important feature of this study was the assessment of current antiviral drugs. The antiviral drugs used during the study period were arbidol, oseltamivir, ribavirin, lopinavir/ritonavir, and $\alpha$ - interferon. The results suggest that arbidol might provide more benefits compared with ribavirin in severe patients treated with monotherapy, but the difference between arbidol and oseltamivir was not significant $(P=0.391)$. At the same time, in severe patients who received combination therapy, the combinations that included arbidol showed better benefits. A multicenter randomized controlled clinical trial published on medRxiv recently showed that patients treated with favipiravir had a better recovery rate $(71.4 \%$ vs. $55.9 \% P=0.0199)$ but more side effects were observed compared with arbidol $[16,18]$. There are multiple antiviral drugs being evaluated and tested in trials currently [19], but before better options can be justified, the use of arbidol might be recommended for its relative safety and effectiveness profile.

Using the MuLBSTA scoring system, 46 (88.5\%) patients were correctly classified as at high-risk for death (score $>12$ ), but only $22(42.3 \%)$ were correctly classified as high-risk (score $\geq 2$ ) when using the CURB-65 scoring system. This suggests that the MuLBSTA scoring system is more effective in the mortality risk assessment of patients with COVID-19 $(P<0.001)$ in the early stage of the disease. This is consistent with a previous study [47]. We speculated that the reason why the MuLBSTA scoring system was more effective is that its scoring criteria (age $\geq 60$, smoking status/smoking cessation history, hypertension history, imaging showing multiple lobar infiltrations, lymphocyte counts $\leq 0.8 \times 10^{9} / \mathrm{L}$, or combined with a bacterial infection) can be achieved and evaluated at the early stage of the disease. On the other hand, the parameters analyzed in CURB-65 may not be elevated in the early stage of the disease in the high-risk population. If necessary, an attempt might be made to lower the 
scoring criteria and set 1 as a cut-off point to improve its sensitivity $(44 / 52,84.6 \%$ vs. $46 / 52,88.5 \%, P=0.566)$. Despite that the 2009 IDSA/ATS guidelines recommended CURB-65 as a severity assessment form for community-acquired pneumonia (CAP) [48], the present study suggests that the MuLBSTA scoring system might be a better assessment tool for COVID-19.

\section{Limitations}

This study has several limitations. First, this was a retrospective study conducted at a single center, with a cohort of 550 patients treated after the arrival of the Hebei medical team, which might not necessarily represent the general population of patients. In addition, the falsenegative rates of current SARS-CoV-19 tests are relatively high and might bias the results. Last but not least, due to the retrospective nature of this study and the lack of diverse drugs in the early stage of the epidemic, the observation of the benefits for different antiviral drugs needs to be further confirmed in future randomized controlled trials.

\section{Conclusion}

In conclusion, age $\geq 60$ years, D-dimer $>0.243 \mu \mathrm{g} / \mathrm{ml}$, and lower OI could help clinicians identify patients with increased probabilities for disease progression early and implement timely medical strategies. Arbidol might have benefits in treating severe patients, but there was only one comparator drug in this study (ribavirin), but the efficacy and safety of drugs for COVID-19 still need to be assessed in future clinical trials.

\section{Abbreviations \\ ARDS: Acute respiratory distress syndrome; MODS: Multiple organ failure; WHO: World Health Organization; CT: Computed tomography; IQR: Interquartile range; Ol: Oxygen index; CAP: Community-acquired pneumonia}

\section{Acknowledgments}

We thank all healthcare professionals for their efforts in helping and taking care of the patients with COVID-19 in Wuhan. We also thank the hospital staff members from The No. 7 Hospital of Wuhan and Zhongnan Hospital of Wuhan University, who gave great support on our daily work, and all the patients and their families who provided their data.

\section{Authors' contributions}

YDY and XWG had the idea for and designed the study. YDY, YL and SWK collected the data. XWG, SWK, YL and HXG analyzed the data. YDY, XWG, SWK drafted the manuscript. YDY, XFG and HXG were involved in patient management and organization work. YDY, XWG and XFG revised the final manuscript. All authors approved the final draft of the manuscript. The corresponding author attests that all listed authors meet authorship criteria and that no others meeting the criteria have been omitted. YDY is the guarantor of the study.

\section{Funding}

This study was funded by grants from the Hebei Province Science and Technology Support Program (20277706D). The research was designed, conducted, analyzed, and interpreted by the authors entirely independently of the funding sources.

\section{Availability of data and materials}

The datasets used and/or analyzed during the current study are available from the corresponding author on reasonable request.

\section{Declarations}

Ethics approval and consent to participate

The study was approved by the Institutional Ethics Board of the No. 7 Hospital of Wuhan and the Second Hospital of Hebei Medical University (Code: 2020-R016). Due to the nature of this retrospective study that used anonymized data, the requirement of obtaining informed consents from the patients was waived by the Institutional Ethics Board.

Consent for publication

Not applicable.

\section{Competing interests}

The authors declare that they have no competing interests.

\section{Author details}

'Department of Respiratory and Critical Care Medicine, The Second Hospital of Hebei Medical University, Shijiazhuang 050000, China. ${ }^{2}$ Department of orthopedics, No. 7 Hospital of Wuhan, Wuhan, China. ${ }^{3}$ Department of Respiratory Medicine, Hebei General Hospital, Shijiazhuang, China.

Received: 2 September 2020 Accepted: 3 June 2021

Published online: 10 June 2021

References

1. Huang C, Wang Y, Li X, Ren L, Zhao J, Hu Y, et al. Clinical features of patients infected with 2019 novel coronavirus in Wuhan, China. Lancet. 2020;395(10223):497-506. https://doi.org/10.1016/S0140-6736(20)30183-5.

2. Chen N, Zhou M, Dong X, Qu J, Gong F, Han Y, et al. Epidemiological and clinical characteristics of 99 cases of 2019 novel coronavirus pneumonia in Wuhan, China: a descriptive study. Lancet. 2020;395(10223):507-13. https:// doi.org/10.1016/S0140-6736(20)30211-7.

3. Wang D, Hu B, Hu C, Zhu F, Liu X, Zhang J, et al. Clinical characteristics of 138 hospitalized patients with 2019 novel coronavirus-infected pneumonia in Wuhan, China. Jama. 2020;323:1061-9.

4. Guan WJ, Ni ZY, Hu Y, Liang WH, Ou CQ, He JX, et al. Clinical Characteristics of Coronavirus Disease 2019 In China. N Engl J Med. 2020;382:1708-20.

5. Zhang JJ, Dong X, Cao YY, Yuan YD, Yang YB, Yan YQ, et al. Clinical characteristics of 140 patients infected with SARS-CoV-2 in Wuhan, China. Allergy. 2020;75(7):1730-41. https://doi.org/10.1111/all.14238.

6. World Health Organization (WHO). Coronavirus disease (COVID-2019) situation reports. https://www.who.int/emergencies/diseases/novel-corona virus-2019/situation-reports. Accessed 20 June 2020.

7. World Health Organization. COVID-19 Weekly Epidemiological Update. October 27, 2020. https://www.who.int/publications/m/item/weeklyepidemiological-update\%2D\%2D-27-october-2020. Accessed 28 Oct 2020

8. WHO. WHO Director-General's opening remarks at the media briefing on COVID-19 - 11 March 2020. https://www.who.int/dg/speeches/detail/whodirector-general-s-opening-remarks-at-the-media-briefing-on-covid-19..-11march-2020. Accessed 20 June 2020.

9. Sanche S, Lin YT, Xu C, Romero-Severson E, Hengartner N, Ke R. High contagiousness and rapid spread of severe acute respiratory syndrome coronavirus 2. Emerg Infect Dis. 2020;26(7):1470-7. https://doi.org/10.3201/ eid2607.200282

10. Wu Z, McGoogan JM. Characteristics of and important lessons from the coronavirus disease 2019 (COVID-19) outbreak in China: summary of a report of 72314 cases from the Chinese center for disease control and prevention. Jama. 2020;323(13):1239-42. https://doi.org/10.1001/jama.2020.2 648.

11. Centers for Disease Control and Prevention. Coronavirus (COVID-19). https:// www-cdc-gov.acces.bibl.ulaval.ca/coronavirus/2019-ncov/index.html. Accessed 20 June 2020.

12. National Health Commission of the People's Republic of China. Chinese management guideline for COVID-19 (version 7.0). http://www.nhc.gov.cn/ xcs/zhengcwj/202003/46c9294a7dfe4cef80dc7f5912eb1989/files/ce3e694 5832a438eaae415350a8ce964.pdf. Accessed 20 June 2020. 
13. World Health Organization. Clinical management of COVID-19: interim guidance. 2020. https://apps.who.int/iris/bitstream/handle/10665/332196/ WHO-2019-nCoV-clinical-2020.5-eng.pdf.

14. U.S. Food \& Drug Adminiatration. FDA New realease. Coronavirus (COVID19) Update: FDA Revokes Emergency Use Authorization for Chloroquine and Hydroxychloroquine. https://www.fda.gov/news-events/press-a nnouncements/coronavirus-covid-19-update-fda-revokes-emergency-use-a uthorization-chloroquine-and. Accessed 20 June 2020.

15. Chowdhury MS, Rathod J, Gernsheimer J. A rapid systematic review of clinical trials utilizing chloroquine and hydroxychloroquine as a treatment for COVID-19. Acad Emerg Med. 2020;27(6):493-504. https://doi.org/1 0.1111/acem.14005.

16. Chen C, Huang JY, Cheng ZS. Favipiravir versus Arbidol for COVID-19: A Randomized Clinical Trial. medRxiv. 2020;2020:03.17.20037432.

17. Lipinski S, Bremer L, Lammers T, Thieme F, Schreiber S, Rosenstiel P. Coagulation and inflammation. Molecular insights and diagnostic implications. Hamostaseologie. 2011;31(02):94-102, 104. https://doi.org/10.54 82/ha-1134.

18. Zhu Z, Lu Z, Xu T, Chen C, Yang G, Zha T, et al. Arbidol monotherapy is superior to lopinavir/ritonavir in treating COVID-19. J Inf Secur. 2020;81:e213.

19. Cao B, Wang Y, Wen D, Liu W, Wang J, Fan G, et al. A trial of Lopinavirritonavir in adults hospitalized with severe Covid-19. N Engl J Med. 2020; 382(19):1787-99. https://doi.org/10.1056/NEJMoa2001282.

20. Beigel JH, Tomashek KM, Dodd LE, Mehta AK, Zingman BS, Kalil AC, et al. Remdesivir for the treatment of Covid-19 - final report. N Engl J Med. 2020; 383(19):1813-26. https://doi.org/10.1056/NEJMoa2007764

21. Doi $Y$, Hibino M, Hase R, Yamamoto M, Kasamatsu Y, Hirose M, et al. A prospective, randomized, open-label trial of early versus late favipiravir in hospitalized patients with COVID-19. Antimicrob Agents Chemother. 2020; 64(12). https://doi.org/10.1128/AAC.01897-20.

22. Alattar R, Ibrahim TBH, Shaar SH, Abdalla S, Shukri K, Daghfal JN, et al. Tocilizumab for the treatment of severe coronavirus disease 2019. J Med Virol. 2020;92(10):2042-49.https://doi.org/10.1002/jmv.25964.

23. Xu X, Han M, Li T, Sun W, Wang D, Fu B, et al. Effective treatment of severe COVID-19 patients with tocilizumab. Proc Natl Acad Sci U S A. 2020;117(20): 10970-5. https://doi.org/10.1073/pnas.2005615117.

24. Garner JS, Jarvis WR, Emori TG, Horan TC, Hughes JM. CDC definitions for nosocomial infections, 1988. Am J Infect Control. 1988;16(3):128-40. https:// doi.org/10.1016/0196-6553(88)90053-3.

25. Definition Task Force ARDS, Ranieri VM, Rubenfeld GD, Thompson BT, Ferguson ND, Caldwell E, et al. Acute respiratory distress syndrome: the Berlin Definition. JAMA. 2012;307(23):2526-33.

26. Khwaja A. KDIGO clinical practice guidelines for acute kidney injury. Nephron Clin Pract. 2012;120(4):c179-84. https://doi.org/10.1159/000339789.

27. Taylor FB Jr, Toh CH, Hoots WK, Wada H, Levi M. Scientific subcommittee on disseminated intravascular coagulation (DIC) of the international society on thrombosis and Haemostasis (ISTH). Towards definition, clinical and laboratory criteria, and a scoring system for disseminated intravascular coagulation. Thromb Haemost. 2001;86(5):1327-30. https://doi.org/10.1055/ s-0037-1616068.

28. Zhou F, Yu T, Du R, Fan G, Liu Y, Liu Z, et al. Clinical course and risk factors for mortality of adult inpatients with COVID-19 in Wuhan, China: a retrospective cohort study. Lancet. 2020;395(10229):1054-62. https://doi. org/10.1016/S0140-6736(20)30566-3.

29. Ye Q, Wang B, Mao J. The pathogenesis and treatment of the 'cytokine Storm' in COVID-19. J Inf Secur. 2020;80:607-13.

30. Tisoncik JR, Korth MJ, Simmons CP, Farrar J, Martin TR, Katze MG. Into the eye of the cytokine storm. Microbiol Mol Biol Rev. 2012;76(1):16-32. https:// doi.org/10.1128/MMBR.05015-11.

31. Xu Z, Shi L, Wang Y, Zhang J, Huang L, Zhang C, et al. Pathological findings of COVID-19 associated with acute respiratory distress syndrome. Lancet Respir Med. 2020;8(4):420-2. https://doi.org/10.1016/S2213-2600(20)30076-X.

32. Yang $X, Y u Y, X u J$, Shu H, Xia J, Liu H, et al. Clinical course and outcomes of critically ill patients with SARS-CoV-2 pneumonia in Wuhan, China: a singlecentered, retrospective, observational study. Lancet Respir Med. 2020;8(5): 475-81. https://doi.org/10.1016/S2213-2600(20)30079-5.

33. Choi KW, Chau TN, Tsang O, Tso E, Chiu MC, Tong WL, et al. Outcomes and prognostic factors in 267 patients with severe acute respiratory syndrome in Hong Kong. Ann Intern Med. 2003;139(9):715-23. https://doi.org/10.7326/ 0003-4819-139-9-200311040-00005.
34. Saad M, Omrani AS, Baig K, Bahloul A, Elzein F, Matin MA, et al. Clinical aspects and outcomes of 70 patients with Middle East respiratory syndrome coronavirus infection: a single-center experience in Saudi Arabia. Int J Infect Dis. 2014;29:301-6. https://doi.org/10.1016/j.ijid.2014.09.003.

35. Majumder MS, Kluberg SA, Mekaru SR, Brownstein JS. Mortality risk factors for Middle East respiratory syndrome outbreak, South Korea, 2015. Emerg Infect Dis. 2015;21(11):2088-90. https://doi.org/10.3201/eid2111.151231.

36. Bikdeli B, Madhavan MV, Gupta A, Jimenez D, Burton JR, Der Nigoghossian C, et al. Pharmacological agents targeting Thromboinflammation in COVID19: review and implications for future research. Thromb Haemost. 2020; 120(7):1004-24. https://doi.org/10.1055/s-0040-1713152.

37. Bikdeli B, Madhavan MV, Jimenez D, Chuich T, Dreyfus I, Driggin E, et al. COVID-19 and thrombotic or thromboembolic disease: implications for prevention, antithrombotic therapy, and follow-up: JACC state-of-the-art review. J Am Coll Cardiol. 2020;75(23):2950-73. https://doi.org/10.1016/j.ja cc.2020.04.031.

38. Moores LK, Tritschler T, Brosnahan S, Carrier M, Collen JF, Doerschug K, et al. Prevention, diagnosis, and treatment of VTE in patients with coronavirus disease 2019: CHEST Guideline and Expert Panel Report. Chest. 2020;158(3): 1143-63. https://doi.org/10.1016/j.chest.2020.05.559.

39. Liu Y, Yang Y, Zhang C, Huang F, Wang F, Yuan J, et al. Clinical and biochemical indexes from 2019-nCoV infected patients linked to viral loads and lung injury. Sci China Life Sci. 2020;63(3):364-74. https://doi.org/10.1 007/s11427-020-1643-8.

40. Wiersinga WJ, Rhodes A, Cheng AC, Peacock SJ, Prescott HC. Pathophysiology, transmission, diagnosis, and treatment of coronavirus disease 2019 (COVID-19): a review. JAMA. 2020;324(8):782-93. https://doi. org/10.1001/jama.2020.12839.

41. Gupta A, Madhavan MV, Sehgal K, Nair N, Mahajan S, Sehrawat TS, et al. Extrapulmonary manifestations of COVID-19. Nat Med. 2020;26(7):1017-32. https://doi.org/10.1038/s41591-020-0968-3.

42. Wu C, Chen X, Cai Y, Xia J, Zhou X, Xu S, et al. Risk factors associated with acute respiratory distress syndrome and death in patients with coronavirus disease 2019 Pneumonia in Wuhan, China. JAMA Intern Med. 2020;180:1-11.

43. Ni YN, Chen G, Sun J, Liang BM, Liang ZA. The effect of corticosteroids on mortality of patients with influenza pneumonia: a systematic review and meta-analysis. Crit Care. 2019;23(1):99. https://doi.org/10.1186/s13054-019-23 95-8.

44. Ye Z, Wang Y, Colunga-Lozano LE, Prasad M, Tangamornsuksan W, Rochwerg B, et al. Efficacy and safety of corticosteroids in COVID-19 based on evidence for COVID-19, other coronavirus infections, influenza, community-acquired pneumonia and acute respiratory distress syndrome: a systematic review and meta-analysis. Cmaj. 2020;192(27):E756-e767. https:// doi.org/10.1503/cmaj.200645.

45. Lee N, Allen Chan KC, Hui DS, Ng EK, Wu A, Chiu RW, et al. Effects of early corticosteroid treatment on plasma SARS-associated coronavirus RNA concentrations in adult patients. J Clin Virol. 2004;31(4):304-9. https://doi. org/10.1016/j.jcv.2004.07.006.

46. Arabi YM, Mandourah Y, Al-Hameed F, Sindi AA, Almekhlafi GA, Hussein MA, et al. Corticosteroid therapy for critically ill patients with Middle East respiratory syndrome. Am J Respir Crit Care Med. 2018;197(6):757-67. https://doi.org/10.1164/rccm.201706-11720C.

47. Guo L, Wei D, Zhang X, Wu Y, Li Q, Zhou M, et al. Clinical features predicting mortality risk in patients with viral pneumonia: the MuLBSTA score. Front Microbiol. 2019;10:2752. https://doi.org/10.3389/fmicb.2019.02 752.

48. Charles PG, Davis JS, Grayson ML. Rocket science and the Infectious Diseases Society of America/American Thoracic Society (IDSA/ATS) guidelines for severe community-acquired pneumonia. Clin Infect Dis. 2009; 48(12):1796; author reply 1796-1797-1796; author reply 1797. https://doi. org/10.1086/599227.

\section{Publisher's Note}

Springer Nature remains neutral with regard to jurisdictional claims in published maps and institutional affiliations. 